\title{
Modelling out-of-area admissions
}

\author{
Lewis W. Paton and Paul A. Tiffin
}

\begin{abstract}
Summary
Out-of-area (OOA) placements occur when patients cannot be admitted to local facilities, which can be extremely stressful for patients and families. Thus, the Department of Health aims to eliminate the need for OOA admissions. Using data from a UK mental health trust we developed a 'virtual mental health ward' to evaluate the potential impact of referral rates and length of stay (LOS) on OOA rates. The results indicated OOA rates were equally sensitive to LOS and referral rate. This suggests that investment in community services that reduce both LOS and referral rates are required to meaningfully reduce OOA admission rates.
\end{abstract}

\section{Declaration of interest}

P.A.T. holds an honorary consultant contract with the Tees, Esk and Wear Valleys NHS Foundation Trust.

\section{Keywords}

Out-of-area admissions; referral rate; length of stay; discrete event simulation.

\section{Copyright and usage}

(c) The Royal College of Psychiatrists 2018
There has been a recent focus on out-of-area (OOA) placements, whereby a patient admitted to their local mental health trust for treatment is sent elsewhere to receive treatment. This is primarily because of lack of local bed availability. This can be traumatic for the patient, who loses contact with their support network of family and friends at a vulnerable time. Furthermore, OOA placements have been found to be two-thirds more expensive than best practice. ${ }^{1}$ For these reasons the Royal College of Psychiatrists aims for OOA placements to be 'minimised', 2 and the Department of Health and Social Care has stated a target of eliminating 'inappropriate' OOA placements by 2020/2021. ${ }^{3}$ Moreover, mental health trusts in England must now report the monthly number of OOA placements. ${ }^{4}$

To reduce OOA placement rates bed availability must be better matched to local need. In-patient care is a complex system, with patients constantly arriving and leaving from a unit of finite capacity. Occupancy rates are also dependent on the capacity of neighbouring hospitals and out-patient services. Increasing bed availability is not always desirable or cost-effective. Over the past two decades, in England, specialised community services, such as 'early intervention in psychosis' (EIP) and 'crisis teams' have been implemented. There is evidence that some of these have reduced mental health bed occupancy via reduced length of stay and/or admission rates. ${ }^{5,6}$ Thus, understanding the relative influence of referral rates and length of stay (LOS) would be valuable in planning which community services to invest in if OOA placements are to be avoided.

\section{Discrete event simulation}

As a complex system, modelling the flow of patients through an in-patient service is not amenable to conventional statistical approaches. However, numerical simulation techniques have previous been used as tools to support the planning of health services. ${ }^{7-9}$ One such simulation approach is discrete event simulation (DES), which models a system (for example a hospital ward) as a sequence of separate events (such as a patient presenting for treatment, a patient being discharged) which occur over a set period of time. DES is commonly used to model systems that can be described in this way, such as queues, manufacturing processes and financial investments. Here we use DES to create a 'virtual ward'. We can change the conditions of the simulation which, in this case, allows us to estimate the number of OOA placements that may occur for varying referral rates and LOS. This report aims to demonstrate the potential of DES in mental health service planning.

\section{Method}

A virtual local in-patient unit was created with a fixed number of beds $(n=20)$. 'Patients' then presented, at any time, requesting admission. If a bed was available they were admitted to the (local) unit and treated for a variable amount of time. Once treatment was complete they were discharged. If no bed was available when a virtual patient required admission they were classed as an OOA placement. Thus, the simulation provided a simplified representation of reality, assuming that bed availability depended only on referral rate and LOS independently.

We assumed referral rate and LOS each followed 'random distributions' that had specified means and variances. ${ }^{10}$ These values could initially be set to those observed in the actual service data provided by the Trust for one general, working-age, mental health ward. LOS is positively skewed (i.e. a small number of patients have unusually long admissions). Thus, we used a log-normal distribution for LOS. We chose an exponential distribution for the referral rate since this distribution describes the length of time between arrivals. We set initial values for the distributions based on observed data and that approximated those OOA rates reported in reality. These values could then be systematically varied so that the impact on the OOA rates could be evaluated. Specifically, by varying one parameter and holding the other constant, we could assess which factor had the largest impact on OOA rate. We then altered both parameters simultaneously to determine what combinations of reductions of LOS and referrals could plausibly achieve lower rates of OOA placements. Each simulation, implemented in the Python package SimPy, was repeated 1000 times and the results averaged. ${ }^{11}$

\section{Results}

Our real-world data showed 529 patients requested treatment at the ward over 851 days, equivalent to 1.61 days per request. The average patient spent $2.5 \log$ (days) on the ward, and $17.2 \%$ of the patients could not be treated on the ward and thus must be treated elsewhere. Informed by the data we set the exponential distribution parameter ('lambda') as 1.61, which describes a process where patients arrive at the hospital on average 1.61 days after the previous patient arrived. We initially chose a log-normal distribution with mean $2.5 \mathrm{log}$ (days) and standard deviation of 1.2. However, this value resulted in the simulation overestimating the OOA rate. Through trial and error we instead chose a mean of $2.2 \log$ (days). This choice, 


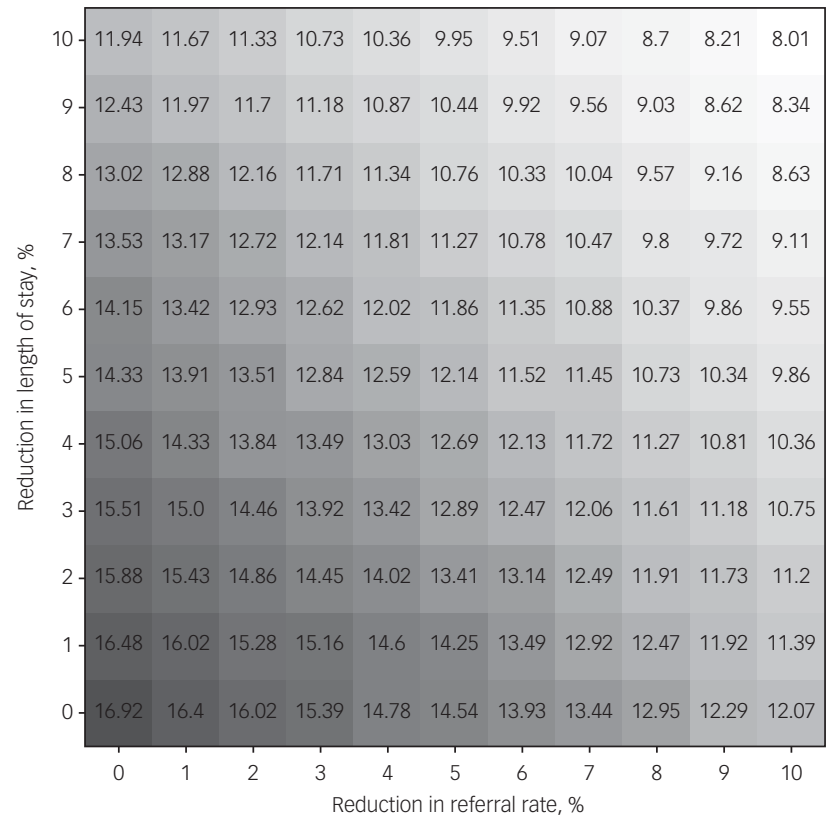

Fig. 1 Out-of-area admission rates from 1000 simulations of the 'virtual' ward for various reductions in referral rate and length of stay.

along with the aforementioned choice of exponential distribution, produced an OOA rate in the simulation of $16.9 \%$ - close to the actual Trust-reported rate at that time of $17.2 \%$.

Figure 1 shows the OOA rates for various values of the two parameters. Simulation showed that, around the initial values of the simulation, small changes in each parameter had a linear impact on the OOA rate. Holding LOS constant and simulating the effect of a $5 \%$ reduction in referral rate gave an OOA rate of $14.5 \%$, a reduction of 2.4 percentage points. Similarly, holding the referral rate constant and simulating the effect of a $5 \%$ reduction in mean LOS gave an OOA rate of $14.3 \%$, a reduction of 2.6 percentage points. Thus, OOA rates appeared to be equally sensitive to referral rate and LOS, over the range of values evaluated.

Figure 1 also allows us to determine which combination of referral rate and LOS may achieve lower OOA placements. For example, an OOA rate in the region of $8.6 \%$ (i.e. half the Trust-reported rate at that time) could be achieved by reducing the referral rate by $8 \%$ and LOS by $10 \%$, or alternatively by reducing the referral rate by $10 \%$ and LOS by $8 \%$.

\section{Discussion}

The results of our simulations suggest that reducing LOS and lowering referral rates have a similar impact on OOA rates. Reducing the amount of time a patient spends on a ward is perhaps more practical - clinically it is not always possible to avoid admission if there are high levels of perceived risk to the patient or others. However, resources can often be coordinated to facilitate a rapid discharge. Integrated models of care, where community services stay involved with patients during an admission, may be effective at reducing LOS (for example in $\mathrm{EIP}^{12}$ ). Indeed, the data we used in this study came from an area with crisis teams and EIP implemented, which will have already had a positive impact on the referral rate and LOS. Nevertheless, our findings suggest that, in the absence of increased in-patient capacity, substantial reductions in both demand and LOS would be needed to achieve OOA placement rates of under $10 \%$.

The presented model is clearly a simplification of the real-world problem. However, the ability to mimic the real-world data suggests that even such a simple simulation has some utility. Modelling an interdependent system of wards and hospitals and the possible dependency between referral rate and LOS would demand a more complex approach, although it is feasible within a DES framework.

Our results suggest that further investing in community services that both reduce LOS (such as EIP) and referral rates (for example crisis teams ${ }^{5}$ ) are likely to be effective in reducing OOA placement rates. We noted that the median LOS in the Trust data used to develop the model was somewhat lower than the national value for 2013-2014 (15 v. 23 days). ${ }^{13}$ This may be because the Trust in question already has well-embedded specialist community services. Moreover, the population served is characterised by relatively low levels of urbanicity. This suggests that services with longer baseline LOS values may be able to achieve somewhat greater reductions in OOA rates via the use of community services. It is also likely that reduced social care provision will lead to delayed discharges in some cases, perhaps predominantly affecting those patients with longer hospital stays. Such influences could be modelled via more complex simulation studies that could guide the optimum allocation of resources at a time of increased financial restraints.

Lewis W. Paton, PhD, Research Fellow, Department of Health Sciences, University of York, UK; Paul A. Tiffin, MD, Reader in Psychometric Epidemiology, Department of Health Sciences, University of York, UK

Correspondence: Lewis W. Paton, Department of Health Sciences, Seebohm Rowntree Building, University of York, Heslington, York Y010 5DD, UK. Email: lewis.paton@york.ac.uk

First received 25 Jan 2018, final revision 24 Apr 2018, accepted 18 May 2018

\section{Funding}

This work was funded by the Tees, Esk and Wear Valleys NHS Foundation Trust.

\section{References}

1 Edwards T, Meier R, Killaspy H. Making the Case for a Rehabilitation Facility. Faculty of Rehabilitation and Social Psychiatry of the Royal College of Psychiatrists, 2010.

2 Edwards T, Wolfson P, Killaspy H. A Guide to Good Practice in the Use of Out-OfArea Placements. Royal College of Psychiatrists' Faculty of Rehabilitation and Social Psychiatry, 2012.

3 Department of Health and Social Care. Out of Area Placements in Mental Health Services for Adults in Acute Inpatient Care. Department of Health and Social Care, 2016 (https://www.gov.uk/government/publications/oaps-in-mentalhealth-services-for-adults-in-acute-inpatient-care/out-of-area-placements-inmental-health-services-for-adults-in-acute-inpatient-care).

4 Blackburn P. Trusts Must Publish OOA Numbers. BMA, 2017. (https://www. bma.org.uk/news/2017/november/trusts-must-publish-o-o-a-numbers).

5 Glover G, Arts G, Babu KS. Crisis resolution/home treatment teams and psychiatric admission rates in England. Br J Psychiatry 2006; 189: 441-5.

6 McCrone P, Singh SP, Knapp M, Smith J, Clark M, Shiers D, et al. The economic impact of early intervention in psychosis services for children and adolescents. Early Interv Psychiatry 2013; 7: 368-73.

7 Zhu Z, Hoon Hen B, Liang Teow K. Estimating ICU bed capacity using discrete event simulation. Int J Health Care Qual Assur 2012; 25: 134-44.

8 Goldman J, Knappenberger HA, Eller J. Evaluating bed allocation policy with computer simulation. Health Serv Res 1968; 3: 1-19.

9 Vassilacopoulos G. A simulation model for bed allocation to hospital inpatient departments. Simulation 1985; 45: 233-41.

10 Forbes C, Evans M, Hastings N, Peacock B. Statistical Distributions. John Wiley and Sons, 2011.

11 Team SimPy. SimPy: Discrete Event Simulation for Python. SimPy, 2017 (https:// simpy.readthedocs.io/en/latest/)

12 Tiffin PA, Hudson S. An early intervention in psychosis service for adolescents. Early Interv Psychiatry 2007; 1: 212-8.

13 Health and Social Care Information Centre. Mental Health Bulletin: Annual Report from MHMDS Returns 2013-14. HSCIC, 2014 (http://digital.nhs.uk/ catalogue/PUB15990). 